\author{
Alterstice \\ Revue internationale de la recherche interculturelle \\ International Journal of Intercultural Research \\ Revista International de la Investigacion Intercultural
}

\title{
L'atelier de situations interculturelles : une méthodologie pour comprendre l'acte à poser en contexte pluriethnique
}

\section{Bob W. White et Danielle Gratton}

Volume 7, numéro 1, 2017

L’interculturel dans la Cité : actes à poser en contexte pluriethnique

URI : https://id.erudit.org/iderudit/1040612ar

DOI : https://doi.org/10.7202/1040612ar

Aller au sommaire du numéro

Éditeur(s)

Alterstice

ISSN

1923-919X (numérique)

Découvrir la revue

Citer cet article

White, B. W. \& Gratton, D. (2017). L’atelier de situations interculturelles : une méthodologie pour comprendre l'acte à poser en contexte pluriethnique.

Alterstice, 7(1), 63-76. https://doi.org/10.7202/1040612ar

\section{Résumé de l'article}

Depuis une dizaine d'années, une série d'évènements donnent l'impression que le Québec cherche à mieux définir son modèle d'intégration basé sur la notion d'interculturalisme. Mais comment définir l'approche interculturelle et comment savoir si elle favorise véritablement l'inclusion des nouveaux arrivants ? Dans le cadre du partenariat de recherche " Vers une ville interculturelle » nous avons organisé une série d'ateliers pratiques afin de faciliter la mise en commun de ce que les différents acteurs du milieu urbain voient comme situations interculturelles problématiques. Les « ateliers de situations interculturelles » ont donné des réponses préliminaires aux trois questions suivantes : 1) Quelles situations problématiques voyons-nous sur le terrain ? 2) Quels outils sont à notre portée pour répondre à ces situations ? 3) Sur quels cadres d'analyse nous appuyons-nous pour orienter nos actions? Pendant cet atelier, qui a été organisé dans différents milieux professionnels et institutionnels, les participants ont travaillé en équipe afin d'identifier et d'analyser un nombre limité de situations qui proviennent de leurs milieux d’intervention respectifs. Les résultats préliminaires des ateliers démontrent la difficulté de distinguer entre les situations interculturelles et les préoccupations par rapport aux situations, mais aussi la difficulté de la centration sur les normes et les valeurs de la société d'accueil. L'analyse de plusieurs contextes d'intervention fait ressortir les différences individuelles et organisationnelles et démontre, entre autres, que les intervenants sont souvent pris entre les exigences contradictoires du multiculturalisme et de l'interculturalisme.
Ce document est protégé par la loi sur le droit d'auteur. L'utilisation des services d’Érudit (y compris la reproduction) est assujettie à sa politique d'utilisation que vous pouvez consulter en ligne.

https://apropos.erudit.org/fr/usagers/politique-dutilisation/ 


\section{7}

ARTICLE THÉMATIQUE

\section{L'atelier de situations interculturelles : une méthodologie pour comprendre l'acte à poser en contexte pluriethnique}

Bob W. White ${ }^{1}$ et Danielle Gratton ${ }^{1,2}$

\section{Résumé}

Depuis une dizaine d'années, une série d'évènements donnent l'impression que le Québec cherche à mieux définir son modèle d'intégration basé sur la notion d'interculturalisme. Mais comment définir l'approche interculturelle et comment savoir si elle favorise véritablement l'inclusion des nouveaux arrivants ? Dans le cadre du partenariat de recherche "Vers une ville interculturelle " nous avons organisé une série d'ateliers pratiques afin de faciliter la mise en commun de ce que les différents acteurs du milieu urbain voient comme situations interculturelles problématiques. Les "ateliers de situations interculturelles» ont donné des réponses préliminaires aux trois questions suivantes : 1) Quelles situations problématiques voyons-nous sur le terrain ? 2) Quels outils sont à notre portée pour répondre à ces situations? 3) Sur quels cadres d'analyse nous appuyons-nous pour orienter nos actions? Pendant cet atelier, qui a été organisé dans différents milieux professionnels et institutionnels, les participants ont travaillé en équipe afin d'identifier et d'analyser un nombre limité de situations qui proviennent de leurs milieux d'intervention respectifs. Les résultats préliminaires des ateliers démontrent la difficulté de distinguer entre les situations interculturelles et les préoccupations par rapport aux situations, mais aussi la difficulté de la centration sur les normes et les valeurs de la société d'accueil. L'analyse de plusieurs contextes d'intervention fait ressortir les différences individuelles et organisationnelles et démontre, entre autres, que les intervenants sont souvent pris entre les exigences contradictoires du multiculturalisme et de l'interculturalisme.

\section{Rattachement des auteurs}

${ }^{1}$ Université de Montréal, Montréal, Canada; ${ }^{2}$ Centre intégré de santé et de services sociaux de Laval (CISSS-Laval), Montréal, Canada

\section{Correspondance}

bob.white@umontreal.ca

\section{Mots clés}

situations interculturelles; acte à poser; compétences interculturelles; cohésion sociale; cadres de référence; interculturalisme; pluralisme

\section{Pour citer cet article}

White, B. W. et Gratton, D. (2017). L'atelier de situations interculturelles : une méthodologie pour comprendre I'acte à poser en contexte pluriethnique. Alterstice, 7(1), 63-76. 


\section{Introduction}

Au Québec comme ailleurs, les changements démographiques récents liés à l'immigration mènent à des situations de mixité inédites, créant autant de possibilités que de peurs (Nail, 2015). S'il est vrai qu'il y a une "diversification de la diversité " dans les métropoles et espaces urbains des pays industrialisés, il est aussi vrai que nous avons besoin d'outils et de concepts plus robustes pour faire l'analyse des interactions en contexte pluriethnique (Amin, 2012; Vertovec, 2007). Les dynamiques dites «interculturelles» sont vécues à plusieurs échelles : à l'intérieur de nos foyers, dans les différents milieux de vie et contextes de travail, à travers nos interactions dans l'espace public et dans les institutions qui sont censées être garantes d'égalité entre citoyens de toutes origines. Un regard critique et un cadre d'analyse systémique nous permettent de voir que les interactions en contexte interculturel, parfois loin de l'idéal de l'harmonie et la compréhension mutuelle, peuvent être aussi une source de tensions sociales et de discrimination à l'égard des minorités ethniques et visibles. Certains types d'interactions contribuent positivement au sentiment d'appartenance des nouveaux arrivants et à la cohésion sociale. D'autres interactions renforcent des perceptions négatives et créent de nouvelles formes de discrimination et d'exclusion. Quelles situations ont le plus d'impact sur la cohésion sociale dans l'espace urbain ? Comment identifier et analyser les situations récurrentes qui composent cette complexité à l'échelle d'un territoire donné ?

Pour répondre à ces questions, nous avons développé une méthodologie qui permet de saisir relativement rapidement les problématiques rencontrées dans différents milieux de pratique dans l'espace urbain : un atelier de recherche-action sur les situations interculturelles. Après quelques considérations sur les notions clés de cet article (notamment " situations » et « interculturel »), nous allons expliquer l'émergence et l'organisation des ateliers que nous avons développés. Ensuite, nous allons présenter la première version d'une grille pour l'analyse des situations interculturelles et proposer quelques pistes de réflexion à partir des constats préliminaires de la première phase de cette recherche. En conclusion, nous allons examiner la possibilité d'analyser cet atelier au-delà de sa contribution méthodologique ou de sa capacité à générer des données, en considérant que le travail du chercheur constitue, lui aussi, un acte à poser. De ce point de vue, les éléments présentés ici visent à la fois l'explicitation de notre démarche méthodologique et une réflexion critique sur son impact en contexte d'intervention.

\section{L'étude des « situations interculturelles »}

Dans la conjoncture historique actuelle - intensification de la mobilité des populations, écart grandissant dans les inégalités entre riches et pauvres, nouvelles formes de violence radicale - les dynamiques interculturelles en contexte urbain constituent une source d'inquiétude. Ces facteurs de changement nous obligent à développer des nouvelles compétences sociales et organisationnelles qui vont au-delà d'un raisonnement purement économique (White, Gratton et et Rocher, 2015), autant pour les citoyens d'ici et d'ailleurs que pour les professionnels qui interviennent auprès des clientèles issues de l'immigration. Sans cadre d'analyse commun, chacun analyse les situations selon son idéologie personnelle, disciplinaire ou politique, et selon des impressions ou des informations partielles, ce qui met de l'huile sur le feu de l'inquiétude (Ahmed, 2012; Maalouf, 1998). La méthodologie que nous proposons mobilise un certain nombre de principes interculturels afin de prendre connaissance des situations auxquelles doivent faire face les intervenants qui travaillent en contexte pluriethnique. Cette méthodologique nous permet ensuite de faire une analyse de l'acte à poser en contexte interculturel, tout en permettant une réflexion critique sur les compétences interculturelles requises, autant pour les intervenants que pour les gestionnaires, et ce, dans différents milieux de pratique ${ }^{1}$.

Commençons par quelques mises en garde terminologiques. Premièrement, le mot « interculturel » est lui-même source de malaises (Rocher et White, 2014; White, 2014). Mal compris et rarement défini, l'interculturel est souvent associé à plusieurs formes de culturalisme, nationalisme et angélisme. Les critiques à l'égard de

\footnotetext{
1 Par « acte à poser " nous voulons faire référence à chaque action faite par des acteurs et en rapport avec des mandats professionnels, organisationnels ou institutionnels et qui, d'un point de vue systémique, pourrait avoir un impact sur la faisabilité et l'efficacité dans les contextes pluriethniques. Selon Bakhtin (1993), l'acte reflète la conscience de l'individu mais relève de l'action morale qui lie le soi à l'Autre. Dans le cas présent, ce lien est en rapport avec les obligations et les mandats institutionnels (Gratton, 2013) ou encore avec les règles du vivre-ensemble dans un milieu donné (White, 2016).
} 
l'interculturel expriment aussi des préoccupations tout à fait valables, par exemple s'assurer que le modèle d'analyse soit apte à prendre en considération les différentes formes de diversité qui coexistent (sans se limiter à l'identité culturelle) et qui s'entrecroisent dans les espaces urbains (Martiniello, 2011; Vertovec 2007). Plusieurs observateurs ont remarqué que certains modèles interculturels ignorent la discrimination qui produit de nouvelles formes d'exclusion et d'inégalité (Cantle, 2012; Labelle, 2010). Malheureusement, la plupart de ces critiques ne tiennent pas compte de la diversité des approches à l'intérieur du courant interculturel et elles ne font pas de distinction entre le champ de recherche interculturel et l'utilisation de ce dernier dans différents milieux de pratique professionnelle (Emongo et White, 2014). En se basant sur des informations partielles, elles véhiculent des perceptions négatives à l'égard d'un champ où, il faut le reconnaître, il manque de normes professionnelles, de foyer disciplinaire et de programmes de formation accrédités.

De plus, une confusion persiste entre deux utilisations du terme, la première faisant référence à une réalité sociale de mixité entre personnes de diverses origines ethnoculturelles - l'interculturalité - et la seconde relevant plutôt du domaine normatif, correspondant à un ensemble de principes et constats qui composerait un modèle de la gestion de la diversité spécifique au Québec - dans ce sens, et par opposition au multiculturalisme canadien, on parle d'interculturalisme. Contrairement à ce que certains proposent (Bouchard, 2012), cette orientation politique ne fait pas l'objet d'un consensus au Québec, et jusqu'à maintenant elle n'a pas fait l'objet d'une politique ou d'une loi officielle (Rocher et White, 2014). Outre la réalité sociale (l'interculturalité) et l'orientation politique (l'interculturalisme), il existe un troisième registre du terme, c'est à dire un courant de pensée et d'action qui permet une réflexion approfondie sur les conditions de la rencontre en contexte pluriethnique. Nous insistons sur ces distinctions, car les fondements épistémologiques derrière les différents registres du terme peuvent avoir un impact sur la façon de concevoir l'acte à poser dans des situations interculturelles qui sont, selon nos recherches, de plus en plus complexes.

Il est difficile d'éviter l'utilisation du substantif (Emongo, 2014) pour définir l'interculturel, du moins au Québec: l'interculturel, c'est la relation entre personnes venant d'horizons culturels différents, mais c'est aussi une méthode d'analyse ou une façon de faire. Cette définition n'est tant exhaustive qu'heuristique, puisqu'elle nous permet de faire la distinction entre les dynamiques sociales en contexte interculturel (interculturalité) et les approches interculturelles qui formeraient un paradigme de recherche ou d'intervention. Nous savons, par exemple, qu'on peut travailler dans un contexte interculturel sans avoir un modèle interculturel et que l'utilisation du terme « interculturel» ne garantit en rien l'utilisation d'un modèle ou d'une approche interculturelle. Nous savons également qu'il y a plusieurs façons de « faire de l'interculturel » et qu'il existe très peu d'espaces institutionnels ou officiels pour réfléchir à la diversité des approches dans ce domaine relativement récent ${ }^{2}$. Alors, comment définir les contours d'une approche interculturelle? Dans le cadre de nos recherches sur les dynamiques interculturelles dans l'espace urbain, nous avons identifié un certain nombre de fils conducteurs ou traits communs qui traversent différentes écoles de pensée sur l'interculturel : 1) une posture d'humilité et de curiosité face aux différentes formes de savoirs venus d'ailleurs, 2) une éthique relationnelle qui met l'accent sur l'aspect coproduit du savoir, 3) un cadre d'analyse interactionniste et systémique qui tient compte de situations et de contextes réels d'interaction, 4) un modèle d'analyse de la communication en trois parties (prise de conscience de la différence, regard mutuel sur l'identité de soi et de l'autre, recherche de significations communes) et enfin 5) un accent mis sur les barrières dans la communication et l'utilisation d'outils qui permettent de réduire ou compenser les écarts de compréhension.

En utilisant l'expression "situations interculturelles" ${ }^{3}$, nous voulons faire référence à un nombre infini d'interactions quotidiennes en contexte pluriethnique où les préjugés et des barrières objectives peuvent devenir

\footnotetext{
${ }^{2}$ L'interculturel comme domaine de recherche existe depuis au moins un demi siècle. Il a été développé dans plusieurs champs disciplinaires (notamment en éducation, psychologie, travail social, gestion et communications) et dans plusieurs contextes régionaux et nationaux. Pour un historique de l'interculturel au Québec, voir Emongo et White (2014).

3 Dans le milieu de l'intervention, la notion de situation renvoie à une unité descriptive sur laquelle au moment de l'analyse on peut appliquer différents modèles disciplinaires. On retrouve ce terme courant notamment dans le programme de formation en relations interculturelles du ministère de la Santé et des services sociaux du Québec (pour en savoir plus, voir Proulx, 1998, et Gratton, 2009).
} 
des obstacles à la communication, à l'efficacité des actes à poser dans l'application des mandats, et se transformer ainsi en facteurs d'exclusion. Cette définition met l'accent sur les obstacles dans la communication, même si les interactions en contexte pluriethnique ne sont pas toujours négatives (Amin, 2012). L'analyse de situations sert, au besoin, à mettre en évidence des problématiques interculturelles récurrentes dont les composantes ne tiennent pas seulement compte de la bonne ou de la mauvaise volonté de chacun, mais aussi des facteurs qui conditionnent l'efficacité des actes à poser" ${ }^{4}$. Déjà en 1992, Legault et Lafrenière utilisaient l'expression "situations d'incompréhension interculturelle " pour décrire les différentes situations de communication problématiques rencontrées par les intervenants travaillant en contexte pluriethnique à Montréal (Legault et Lafrenière, 1992). Dans le modèle de Cohen-Émérique (1993), dont les écrits sur l'intervention en contexte interculturel ont eu beaucoup d'impact au Québec, la notion d'« incident critique » désigne une situation communicationnelle entre personnes d'origines diverses qui fait ressortir des " chocs culturels ». Cohen-Émérique (2011, p. 439) indique que la méthode des chocs culturels peut aussi être nommée "la méthode des incidents critiques " : pour elle, cette méthode permet aux participants à ses formations de narrer « une interaction limitée qui se déroule dans un temps et un lieu donnés ". Dans ce cadre d'analyse, l'interprétation reste au niveau individuel et ne s'intéresse pas aux barrières systémiques qui peuvent avoir un impact sur l'efficacité des actes à poser et qui renvoient à des compétences interculturelles organisationnelles nécessaires pour fournir les nouvelles conditions exigées par l'intervention en contexte pluriethnique.

L'approche de Cohen-Émérique consiste à identifier un certain nombre de situations utilisées pour des besoins d'apprentissage, une approche qui a été reprise par plusieurs chercheurs et experts interculturels dans le domaine de l'intervention au Québec ${ }^{5}$. Selon Roy (1992), les incidents critiques sont aussi des situations qui se reproduisent de façon récurrente et dont la résolution nécessite une expertise interculturelle. Roy reprend ainsi la notion d'incident critique de Cohen-Émérique, mais elle en propose une autre utilisation : l'incident critique devient une récurrence indiquant une difficulté contextuelle interculturelle qui demande une attention particulière pour sa résolution. On le voit, comme souvent en interculturel, la notion d'incident critique a sa propre généalogie, un sujet qui mérite une analyse en soi ${ }^{6}$.

Dans le cadre de nos ateliers nous avons constaté que cette utilisation des incidents critiques représente des limites dans l'analyse et dans la recherche de solutions, non seulement parce que (comme nous allons voir en bas) la capacité d'identifier et de décrire les situations interculturelles fait partie des compétences à développer en contexte d'intervention, ce que Cohen-Émérique (2011) constate aussi. Le fait de travailler avec des situations établies à l'avance met les participants dans un rôle passif d'apprentissage. De plus cette approche peut avoir l'effet de réduire la grande diversité d'actes à poser qui préoccupent les participants à l'atelier, ce qui n'aide pas à bien cerner les problèmes contextuels importants d'un milieu à l'autre ${ }^{7}$.

La méthodologie que nous avons développée dans nos ateliers de situations s'inspire de plusieurs sources, non seulement de Roy et Cohen-Émérique, mais aussi de Das (1993). Ces auteurs ont eu une influence importante dans les travaux faits dans le cadre d'un projet de recherche-action mené par l'Agence de la santé et des services sociaux

\footnotetext{
${ }^{4}$ En 1951 l'anthropologue Gregory Bateson a écrit que l'analyse de la communication commence par une " situation sociale ». II faut noter que Bateson a aussi travaillé avec des intervenants (Bateson et Reusch, 1951). Son parcours intellectuel, qui a eu beaucoup d'impact sur les approches systémiques dans les sciences humaines, met en évidence comment deux modèles disciplinaires peuvent s'appliquer à l'analyse d'une même situation.

${ }^{5}$ Danielle Gratton, communication personnelle.

${ }^{6}$ Selon plusieurs sources, la source de l'expression serait l'anthropologie physique de la fin du $19^{e}$ siècle. Le terme sera repris dans les années 1950 par des chercheurs en psychologie sociale (notamment Flanagan) avant d'être récupéré par les spécialistes de la communication interculturelle dans les milieux professionnels de la santé, de la gestion, et de l'éducation internationale (Bathurst et LaBrack, 2012).

${ }^{7}$ Dans le cadre de nos ateliers de recherche-action, les intervenants et les gestionnaires nous rappellent, avec raison, que les relations interculturelles ne sont pas toujours sources de tension ou de conflit et que la terminologie que nous utilisons doit être en mesure de capter le plus grand nombre possible de situations. Ainsi, pour certains participants, l'expression « incident critique " semblait trop négatif.
} 
de Montréal-Centre dans les années $2000^{8}$. Ces travaux, réalisés avec une méthodologie rigoureuse et une évaluation systématique sur plusieurs années, ont permis aux chercheurs et intervenants impliqués dans la recherche de faire plusieurs constats. ${ }^{9}$ Premièrement, ils ont démontré que, même lors de formations, il est avantageux d'utiliser un modèle recherche-action dans lequel les participants sont considérés comme des experts de leur milieu, car ils connaissent les dynamiques interculturelles dans lesquelles ils sont impliqués, et parfois ils ont développé des expertises spécifiques au milieu d'intervention. Leur participation dans l'identification de situations concrètes permet de valider la pertinence du contenu interculturel présenté et renforce également la compréhension par les organisateurs de la formation de leurs réalités pluriethniques. Deuxièmement, ils ont constaté que l'utilisation d'un cadre d'analyse systémique permet d'avoir une meilleure compréhension de l'acte à poser en contexte interculturel, en partie parce que cette approche permet d'expliquer les liens entre les mandats institutionnels, les codes communicationnels et les dynamiques d'inclusion (White, Gratton et Rocher, 2015) ${ }^{10}$. Troisièmement, ces travaux ont permis de dégager l'importance d'appliquer non seulement une expertise interculturelle individuelle, mais aussi une expertise interculturelle organisationnelle dans l'identification et dans l'analyse des situations pluriethniques (Gratton, 2013).

\section{Contexte et organisation de l'Atelier de situations interculturelles}

La méthodologie que nous avons développée sur les situations interculturelles dépend des liens de proximité entre les chercheurs et les différents acteurs et organismes qui travaillent directement auprès des clientèles immigrantes ${ }^{11}$. La collecte de données se fait en concertation avec les organismes membres du partenariat de recherche que nous avons créé et un certain nombre de "tables de concertation " spécialisées en relations interculturelles. Les tables de concertation (organisées par territoire ou par secteur dans le cadre des ententes ministérielles au Québec depuis les années 1990, voir Fourot, 2013) regroupent des intervenants et des organismes issus de milieux divers comme l'éducation, la santé, les services sociaux et l'emploi. La collecte de données s'effectue principalement à partir d'une série d'ateliers de recherche-action ${ }^{12}$. Ces ateliers permettent un repérage de données plus ciblées que ce que permet le travail de terrain traditionnel, en grande partie parce que les participants possédant une connaissance approfondie des dynamiques interculturelles dans leurs secteurs et territoires respectifs, ils jouent le rôle d' "informateur-clés" dans le modèle de la recherche ethnographique. L'atelier permet de poser un regard commun sur les situations et d'identifier un nombre important de situations vécues par tous, puis de réfléchir ensemble sur le cadre partagé qui devrait guider l'intervention.

\footnotetext{
${ }^{8}$ Gratton (2013) explique la présence d'au moins deux courants interculturels au Québec (dont un serait inspiré des travaux de Das et l'autre de Cohen-Émérique). Malgré les similitudes entre les deux approches, des différences importantes demeurent entre elles, notamment en ce qui concerne l'utilisation des termes « centration » et " décentration ». Selon Gratton, I'utilisation de ces deux termes reflète deux traditions de pensée interculturelle et deux idéologies distinctes par rapport à l'acte à poser. Das fait sauter la division entre sociologie pour les sociétés occidentales et anthropologie pour les autres sociétés, appliquant les principes de l'anthropologie aux sociétés occidentales. La centration devient alors un moyen pour prendre conscience de l'enracinement culturel des institutions occidentales et des pratiques et, tout en ayant ses limites, elle offre de nouveaux moyens à adapter en contexte pluriethnique.

${ }^{9}$ Cette évaluation a servi à adapter le contenu du programme cadre de formation interculturelle du ministère de la Santé et des Services sociaux du Québec. Le lecteur peut trouver le contenu développé partir de cette évaluation dans Gratton (2009) ainsi que quelques questions utilisées pour cette évaluation (voir p. 236).

10 II existe plusieurs écoles de pensée sur les approches systémiques. Étant donné l'accent qu'il a mis sur la communication et sur le contexte, nous nous inspirons de l'approche l'école de Palo Alto (voir Gratton, 2013, et White, 2017b).

${ }^{11}$ Avec le soutien du Conseil de recherche en sciences humaines, le projet de recherche "Vers une ville interculturelle " a été financé pour élaborer un programme de recherche partenarial sur les dynamiques interculturelles dans l'espace urbain. Le partenariat est composé d'une douzaine de partenaires venant de trois secteurs d'activité : acteurs communautaires, acteurs municipaux et acteurs universitaires. Cette organisation nous permet de concentrer nos efforts sur les méthodologies et les problématiques interculturelles à l'échelle municipale, sans subir les contraintes qui proviennent d'une approche sectorielle (par exemple le secteur de l'éducation, de la santé, de l'économie, etc.). Pour plus d'information au sujet de partenariat, voir www.villeinterculturelle.net

${ }^{12}$ Tous les participants ont signé des formulaires de consentement conformément aux indications du certificat d'éthique de recherche CERFAS-2012-13-047-D délivré par I'Université de Montréal.
} 
Cette méthodologie a été validée auprès d'environ 200 intervenants travaillant dans des contextes pluriethniques dans la grande région métropolitaine de Montréal. II nous paraît important de souligner ce fait, afin de reconnaître la contribution des différents individus et organismes qui ont participé à la validation de cette approche. Nos ateliers regroupent généralement entre 15 et 25 personnes qui travaillent dans le même contexte organisationnel ou dans des organismes différents mais avec un mandat similaire auprès des clientèles immigrantes sur un même territoire. On recueille les situations décrites afin de s'assurer de la pertinence des outils interculturels disponibles pour les intervenants et la population desservie par les intervenants (voir la section « Pistes d'avenir »).

Animé par deux présentateurs, l'un qui se centre sur le contenu théorique et l'autre qui présente les aspects pratiques, l'atelier vise à identifier et décrire des situations interculturelles afin de mieux comprendre les liens entre les dynamiques interculturelles et la cohésion sociale dans l'espace urbain à Montréal. Cette réflexion commence par trois questions : 1) Est-ce que nous voyons les mêmes situations ? 2) Est-ce que nous avons les mêmes façons de répondre à ces situations ? 3) Est-ce que nous nous appuyons sur un cadre d'analyse commun ?

Pendant le déroulement des ateliers, trois objectifs spécifiques sont visés :

- Se donner une définition opérationnelle de l'approche interculturelle

- Distinguer entre une " situation interculturelle » et une " préoccupation interculturelle »

- Identifier un certain nombre de situations interculturelles concrètes

Pour répondre au premier objectif, nous présentons un contenu permettant aux participants d'avoir un vocabulaire commun sur les différentes formes de diversité et sur les dynamiques de communication en contexte pluriethnique. À cette fin, nous faisons un retour sur les similitudes et les différences entre le multiculturalisme canadien et l'interculturalisme québécois, deux postures pluralistes qui coexistent au Québec, et ce, malgré des différences philosophiques et politiques entre ces deux modèles (Rocher et White, 2014). Cela permet aux participants de comprendre, comme nous l'avons vu au début de cet article, qu'il y a non seulement une confusion entre des phénomènes sociaux (multiculturalité, interculturalité) et des idéologies politiques (multiculturalisme et interculturalisme), mais aussi que la perception des différences entre ces deux modèles correspond souvent à un clivage idéologique entre des orientations fédéralistes et souverainistes.

Cette étape aide à comprendre que les modèles multiculturaliste et interculturaliste partagent une vision pluraliste et qu'au Québec, il y a au moins trois grands courants de pensée pluraliste qui orientent les actions et les politiques : la reconnaissance de la diversité, la lutte contre la discrimination et la recherche du rapprochement par le dialogue ${ }^{13}$. Cette compréhension aide les participants à visualiser la pertinence de chacun de ces courants, tout en appréciant leurs apports et leurs limites. Finalement, nous présentons plusieurs concepts qui permettent de mieux comprendre la spécificité des phénomènes interculturels : les parcours de migration, les différents niveaux de l'identité (individuelle, groupale, humaine) et les mécanismes d'exclusion (stéréotypes, discrimination, racisme, etc.). Une attention particulière est accordée au fonctionnement des préjugés, à partir d'un point de vue herméneutique (White, 2017a) mettant l'accent sur des techniques d'explicitation qui permettent de mobiliser les préjugés dans la lutte contre la discrimination.

Les ateliers durent entre trois et quatre heures, mais souvent les participants estiment que les ateliers devraient durer plus longtemps. Ceci s'explique en partie par le fait que l'analyse des situations interculturelles fait ressortir les différentes positions idéologiques autour de l'acte à poser et que la recherche d'un langage partagé exige plus de temps (White, 2017a). L'utilisation d'un cadre commun permet de mieux focaliser l'attention des participants sur notre deuxième objectif, qui est d'identifier et de décrire des situations interculturelles dont certaines peuvent être problématiques et récurrentes. Le fait de réfléchir ensemble sur ces différentes postures épistémologiques permet aux participants de s'approprier un langage commun fonctionnel afin de faciliter la communication.

\footnotetext{
${ }^{13}$ Pour une explication plus détaillée de ce modèle, voir White (2017b).
} 
Dans le cadre des ateliers, nous utilisons deux outils pour nous aider dans la description des situations interculturelles apportées par les participants. Le premier est un outil de centration (inspirée par Das, 1993) qui permet aux participants de réaliser une prise de conscience sur l'influence de leurs propres appartenances et de leurs préjugés possibles émergeant en milieu pluriethnique. Le deuxième outil est une fiche (inspirée de Gratton, 2009) servant à décrire de la façon la plus neutre possible une situation interculturelle récurrente. II s'agit pour la personne de raconter des situations réelles à partir de ses propres expériences comme professionnel, ensemble avec des pairs ou des collègues, tout en décrivant l'acte à poser en contexte d'interaction. II s'agit ensuite de faire la distinction entre la situation décrite et les préoccupations engendrées par cette situation, une étape importante pour permettre une réflexion sur la présence ou non de préjugés dans la description.

Après quelques explications sur l'utilisation de la fiche de description, les participants travaillent en petits groupes de 3 à 5 personnes. Les animateurs de l'atelier circulent pour offrir de l'aide aux participants qui auraient de la difficulté à comprendre les termes ou le fonctionnement de l'exercice. Certains participants fournissent des exemples de "situations " qui décrivent des conditions de vie sans faire référence à des contextes d'interaction (par exemple les conditions d'insalubrité dans les logements à haute densité d'immigrants, ou des taux de chômage élevés pour les femmes de certaines communautés d'immigration). Dans les cas où les situations présentées ne correspondent pas aux critères spécifiés dans le cadre de l'atelier ${ }^{14}$, les animateurs demandent aux participants de préciser ou de reformuler leur description. Enfin, chaque groupe de travail choisit l'exemple qui sera analysé lors de la recherche de solutions qui se fera en plénière. Outre cet exemple choisi par chaque groupe, chaque participant remplit une fiche individuelle avec un exemple de situation et des informations relatives à cette situation. Les fiches de situations sont anonymes et les participants sont invités, s'ils le veulent, à les remettre aux animateurs à la fin de l'atelier. Ils sont informés que leur fiche fera alors partie d'une collecte de données générale sur les situations interculturelles retrouvées dans différents milieux de pratique dans la grande région de Montréal ${ }^{15}$.

La démarche que nous proposons dans le cadre de l'atelier des situations permet de générer un nombre important de données de terrain dans une diversité de contextes d'intervention. Les situations recueillies varient en fonction du milieu professionnel, du milieu de vie, du territoire, de la période, du moment de l'année et d'autres facteurs qui ont été identifiés comme indicateurs dans l'analyse des situations ${ }^{16}$. La méthodologie développée dans le cadre de cet atelier constitue à la fois un outil pour générer des données et l'esquisse d'un modèle d'analyse pour identifier des pistes de solutions. Un aperçu du modèle d'analyse interculturel que nous utilisons dans le cadre des ateliers de situations interculturelles est présenté dans l'encadré de la page suivante.

À une plus grande échelle, cette technique de recueil de données permet de voir si certaines situations ou certains types de situations traversent des territoires ou des secteurs d'activité. Alnsi, des données sur les situations interculturelles pourraient être utilisées pour connaître les difficultés relatives à certains actes posés dans différents contextes professionnels et dans différents milieux d'intervention. Malgré le fait que la collecte de données ne soit pas encore terminée, nous avons déjà pu faire quelques constats. Premièrement, beaucoup de situations rapportées relèvent des dynamiques d'interaction dans les services communautaires ou publics ${ }^{17}$. Deuxièmement, il y a une prépondérance de situations liées aux questions de langue (surtout à propos de I'utilisation du français ou de sa méconnaissance, ce qui entraîne des problèmes de compréhension), aux rapports

\footnotetext{
14 Pour faciliter le travail dans les ateliers nous avons identifié trois critères pour le choix de situations interculturelles : 1) interactions entre personnes d'origines différentes, 2) communication autour de la cohabitation en contexte pluriethnique et 3) enjeux qui peuvent avoir un impact sur la cohésion sociale.

${ }^{15}$ Cette collecte de données va contribuer au développement d'une plateforme numérique pour l'étude et la recherche sur les situations en contexte interculturel.

16 Dans la première phase des travaux, nous avons identifié une dizaine de dimensions d'analyse (par exemple type d'interaction, contexte de l'interaction, structures dans l'interaction, barrières à la communication, etc.) avec des indicateurs qui sont spécifiques à chaque dimension. Cette liste d'indicateurs sera révisée dans la deuxième phase de recherche.

${ }^{17}$ Ceci pourrait être expliqué par le fait que la plupart de nos ateliers jusqu'à maintenant ont été organisés avec la participation de professionnels venant des milieux communautaires et institutionnels qui travaillent dans le soutien et l'intégration des populations immigrantes. Dans la deuxième phase de la recherche, nous avons prévu cet atelier dans un plus grand nombre de contextes et milieux de pratique.
} 
hommes-femmes et aux pratiques religieuses, trois sujets qui reviennent souvent dans la recherche sur les dynamiques interculturelles. Troisièmement, nous avons constaté que certaines catégories de situations sont plus difficiles à décrire et surtout à analyser. Par exemple, plusieurs participants ont recensé des situations indiquant un manque de participation ou une absence de la part de certaines communautés immigrantes, alors que les services proposés se veulent accessibles à tous.

\title{
Modèle d'analyse interculturel
}

\author{
Étape 1 - Description de la situation \\ Dans des termes le plus neutres possible, on décrit une situation préoccupante. Cette description doit \\ fournir des éléments aptes à donner une image claire de cette situation à quelqu'un qui n'est pas familier \\ du contexte : Quoi ? Qui ? Quand ? Comment ?
}

\section{Étape 2 - Description de la préoccupation}

Pour analyser la situation choisie, il est important de distinguer entre la situation elle-même et la préoccupation qu'elle soulève. Ainsi, suite à l'analyse, on pourra déterminer si la préoccupation est fondée ou non.

\section{Étape 3 - Exercice de centration}

Plusieurs facteurs peuvent avoir un impact sur l'analyse des situations interculturelles. Ces facteurs renvoient, en premier lieu, aux caractéristiques socioculturelles de celui ou de celle qui doit agir sur une situation (par exemple, le mandat de la personne qui décrit la situation, l'arrondissement ou le service où elle travaille, sa formation, son pays d'origine, etc.). Les individus tout comme les organisations doivent se centrer sur les perceptions qui sont liés à leur groupe et leurs propres façons de fonctionner afin de faciliter la comparaison avec les façons de fonctionner qui viennent d'ailleurs.

\section{Étape 4 - Identification des invariants}

Un invariant est un phénomène qui existe dans toutes les sociétés, mais dont l'expression varie d'une société à l'autre. Par exemple, toutes les sociétés humaines se demandent ce qui advient après la mort, mais la réponse à cette question varie selon chaque groupe humain ainsi que, dans certains cas, selon les individus à l'intérieur du groupe. L'identification d'un invariant permet de reconnaître notre humanité commune, pour passer ensuite au niveau des différences groupales, ce qui permet une explicitation des similitudes et des différences entre groupes humains.

\section{Étape 5 - Recherche de solutions}

Les solutions proposées par les animateurs doivent toujours s'appuyer sur le fonctionnement, les rôles et les mandats organisationnels. Clarifier ces questions organisationnelles permet déjà de savoir sur quoi se fonde la prise de décision au sein d'un organisme et de l'expliquer aux personnes concernées. D'un point de vue interculturel, quand une solution a été trouvée, il est important de la documenter en expliquant les raisons de ce choix (mandats, règles et règlements, ressources existantes, etc.). II est également important de documenter cette information car un cumul de situations similaires pourrait amener l'organisation à revoir ultérieurement ses orientations et à transférer ces nouvelles connaissances dans d'autres contextes. 
Dans le cadre de cet article, nous ne pouvons pas donner beaucoup de détails sur les situations interculturelles recensées, sujet que nous allons traiter dans d'autres publications. Cependant, voici quelques exemples généraux de situations qui ont été identifiées et analysées dans le cadre de nos ateliers :

- utilisation des espaces communs pour des activités religieuses comme la prière,

- malaises de personnes (d'ici ou d'ailleurs) qui reçoivent des services par des personnes d'une autre origine,

- communication dans une langue autre que le français dans les services publics,

- soutien financier ou symbolique des activités des associations culturelles monoethniques,

- codes vestimentaires dans les services publics ou dans les services de loisirs.

L'objectif de cet article est davantage de réfléchir sur un dispositif méthodologique qui permet de mieux cerner les nouvelles situations interculturelles auxquelles tout un chacun est confronté en contexte pluriethnique. Nous pensons qu'il sera plus facile d'atteindre ces objectifs en se centrant sur les actes à poser en contexte d'intervention. Nous avons maintenant quelques idées sur les types de situations et les préoccupations de certaines catégories d'acteurs, mais nous ne sommes qu'au début de notre processus de recherche en ce qui concerne l'analyse des situations interculturelles. Ces résultats préliminaires nous servent pour l'instant comme éléments de validation par rapport à la pertinence des ateliers, à leur fonctionnement et leur organisation. Une analyse préliminaire des données nous permet de penser que cette méthodologie offre un potentiel important pour fournir des réponses fiables et crédibles au sujet des interactions qui sont sources de tension ou de préoccupation à l'échelle municipale. De plus, les participants nous indiquent que ce mode de recueil de données leur est utile car cela leur permet de développer un regard commun et leur offre un modèle efficace pour comprendre, du moins en partie, les phénomènes interculturels qu'ils observent dans le contexte de leur travail. Ce dispositif de recherche est en évolution, comme tout outil qui vise à répondre aux besoins des acteurs du terrain. Ceci dit, nous en savons déjà assez pour pouvoir faire quelques constats généraux sur le fonctionnement des ateliers et sur l'utilisation des données générées.

\section{Pistes d'analyse pour l'avenir}

Même si l'objectif principal des ateliers de situations interculturelles est de générer des données sur les dynamiques interculturelles dans le contexte urbain de Montréal, la mise en commun du savoir sur l'acte à poser permet aux participants d'avoir une meilleure compréhension des dynamiques interculturelles de façon plus large. Les ateliers répondent aux besoins de certains acteurs qui sont à la recherche de nouveaux outils pour intervenir de façon plus efficace en contexte interculturel. Nous avons souvent observé que les ateliers créent un effet de soulagement par rapport à la lourdeur de certaines problématiques interculturelles. Dans certains contextes, les ateliers peuvent avoir un impact de rapprochement entre différents acteurs de terrain, ce qui inclut les dynamiques entre ou avec les chercheurs (White, 2017b). Nous n'avons pas fait l'évaluation systématique des facteurs organisationnels ou subjectifs en cause, mais les évaluations réalisées à la fin de chaque atelier indiquent que cette activité a un impact positif sur la collaboration entre les participants et sur la perception de leurs capacités à intervenir en contexte pluriethnique. Ceci dit, même si les participants nous disent que les ateliers permettent un certain apprentissage (concepts, terminologies, utilisation d'outils d'analyse), nous insistons sur le fait que les ateliers de situations interculturelles ne doivent pas être confondus avec des formations sur les compétences interculturelles, qui exigent des conditions particulières pour être efficaces au niveau organisationnel ${ }^{18}$.

Les participants donnent leur consentement à participer aux ateliers de façon anonyme et cette participation permet la collecte de données inédites qui reflètent la réalité des différents acteurs sur le terrain. En échange, les participants ont la possibilité d'approfondir leur compréhension des dynamiques interculturelles et de trouver quelques pistes de solutions pour des problèmes récurrents liés à l'acte à poser dans leurs milieux respectifs. II est important d'insister sur le fait qu'il n'y a pas de solutions miracles ou "recettes " dans l'analyse des situations interculturelles. II y a, par contre, des principes et des outils interculturels qui permettent de faire des analyses des

\footnotetext{
18 II n'est pas toujours facile de distinguer entre la recherche et la formation interculturelle, pourtant deux domaines qui sont marquées par des fortes différences historiques, conceptuelles et épistémologiques (Bathurst, 2015).
} 
dynamiques interculturelles sans tomber dans le culturalisme ou dans l'inaction de réflexion théorique. Il est important de souligner que la collecte de données sur les situations interculturelles, travail qui se fait sur le terrain et qui nécessite la participation de plusieurs catégories de professionnels, va à l'encontre d'une tendance économiste qui cherche à expliquer les problèmes de l'intégration par l'utilisation de données statistiques et à partir d'un certain nombre d'indicateurs en rapport à la participation sur le marché d'emploi (Frozzini et Gratton, 2015) ou à l'accès aux services publics (Côté, Frozzini et Gratton, 2013). L'une des prémisses de ce projet de recherche est effectivement qu'il nous manque des données systématiques fiables (qualitatives et quantitatives) sur les interactions entre les différents groupes humains qui partagent un même territoire municipal. C'est en ce sens que l'idée des « situations interculturelles » prend toute son importance.

Si cette recherche-action répond aux besoins des intervenants et des gestionnaires qui doivent composer avec une multitude d'actes à poser dans un contexte socioéconomique de plus en plus diversifié, il pourrait également avoir un impact (direct ou indirect) sur les programmes et les politiques mobilisés au nom de la cohésion sociale en contexte de migration. La participation aux ateliers permet aux acteurs de différents milieux de prendre de la distance face à chaque acte à poser en contexte pluriethnique, pour en faire un objet de réflexion critique. La majorité des participants aux ateliers ont fait des commentaires positifs par rapport à cet aspect de l'atelier. En ce sens, l'atelier de situations interculturelles constitue en lui-même un acte à poser, puisqu'il est de l'ordre de la recherche-action qui doit s'assurer d'avoir les conditions pour générer des données significatives, ce qui est souvent un défi en contexte pluriethnique. En considérant la méthodologie de l'atelier comme un acte à poser, nous pouvons voir la porosité des frontières entre la recherche et l'action ainsi que les ponts entre les différentes formes de savoir (universitaire, communautaire, municipale, etc.) qui constituent le champ en expansion de l'action interculturelle.

Outre ces retombées générales, nous avons aussi pu faire un certain nombre de constats préliminaires quant aux résultats de recherche générés dans le cadre de ces ateliers. Ces constats relevant d'observations informelles mais récurrentes lors des ateliers, il est utile d'en reprendre un certain nombre (sans ordre précis) comme hypothèses de travail et d'analyses à approfondir dans les recherches futures :

1. La discussion sur les situations, surtout celles qui sont sources de malaises ou de tensions, font ressortir les différentes idéologies personnelles, professionnelles et institutionnelles qui entourent l'acte à poser. Pour prendre un exemple, nous avons pu observer combien sont forts les tabous entourant l'idée de nommer les différences entre le groupe majoritaire et les différents groupes minoritaires. Étant donné la nature délicate de certaines situations, il est important de veiller sur la composition des groupes qui participent à l'atelier et de trouver un cadre commun (même temporaire) pour permettre des échanges de fond sur les situations et les pistes de solutions proposées. Dans certains contextes, des dynamiques de pouvoir organisationnels internes peuvent paralyser le fonctionnement de l'atelier.

2. Certaines situations moins fréquentes, comme des peurs entourant des situations hypothétiques, peuvent avoir autant d'impact sur la cohésion sociale que des situations récurrentes. La recherche sur les situations interculturelles devrait être capable d'expliquer pourquoi certaines catégories de situations sont plus anxiogènes que d'autres et comment concrètement la médiatisation de ces situations est susceptible d'avoir un impact sur la cohésion sociale.

3. Nous avons souvent entendu la question suivante: "Jusqu'où devrait-on adapter nos services ? ". Malgré la ressemblance apparente entre cette préoccupation et celle liée à la notion d'accommodements raisonnables, nous avons relevé moins d'intérêt pour les accommodements $q^{\prime}$ 'on aurait pu le penser au début de la recherche. De plus, nous savons qu'il s'agit d'un dispositif juridique pour régler les situations de conflit et non pas d'un modèle général pour l'analyse des dynamiques en contexte interculturel. Certains participants ont exprimé des réticences envers la notion d'accommodements raisonnables, qui semble mettre trop l'accent sur les cadres de référence et les normes du groupe majoritaire, surtout dans le contexte des relations entre le Canada et le Québec (Rocher et White, 2014). 
4. Nous avons noté beaucoup de confusion entre les approches multiculturelles et les approches interculturelles. Les différents acteurs du terrain se trouvent souvent coincés entre les impératifs contradictoires de ces approches, qui d'un point de vue systémique relèvent d'échelles et de niveaux logiques différents: la reconnaissance dans le modèle multiculturel et le rapprochement ou la promotion des interactions dans le modèle interculturel. D'après nos observations, les milieux francophones à Montréal ressentent beaucoup de malaises face à l'idée du multiculturalisme. Ce malaise (voire préjugé ?) s'exprime autour d'une série de craintes face au communautarisme ou la "ghettoïsation » des communautés linguistiques et culturelles et la création de "vies parallèles » (Cantle, 2012).

5. Nous avons relevé une difficulté généralisée à distinguer entre les situations interculturelles et les préoccupations en rapport avec ces situations, surtout pour ceux qui n'interviennent pas directement sur le terrain (un phénomène discuté par Gratton, 2013). Cette observation pourrait expliquer la frustration des intervenants, pour qui plusieurs programmes ne correspondent pas aux réalités de terrain.

6. Une certaine confusion demeure entre l'utilisation des termes " centration » et " décentration », deux termes souvent associés aux techniques de développement de compétences interculturelles, surtout dans les milieux francophones. Ces deux utilisations, presqu'opposés sur le plan sémantique (se rapprocher ou s'éloigner des traditions dont nous sommes porteurs), visent la même finalité l'explicitation des cadres de référence du soi afin de réduire l'impact des préjugés - mais se réfèrent à deux démarches analytiques et à des idéologies distinctes.

7. Il existe une forte tendance à mettre l'accent sur les compétences individuelles, ce qui laisse les individus et les employés livrés à eux-mêmes au lieu que soient exigés des ajustements dans le fonctionnement des institutions ou des organismes. Selon nos analyses, cette tendance résulte de plusieurs décennies de campagnes de sensibilisation et de formations sur la diversité qui mettent l'accent sur les attitudes et perceptions individuelles en laissant de côté les conditions fournies (ou non) par les organisations et les institutions pour faciliter les interactions et l'efficacité des actes à poser (White, Gratton et Rocher, 2015).

8. Les ateliers ont confirmé certaines de nos hypothèses quant à la proximité entre les approches citoyennes et les approches interculturelles. Les approches interculturelles mettent beaucoup l'accent sur la recherche d'identités partagées en dehors des identités culturelles sans pour autant gommer les différences au nom d'une appartenance citoyenne (White, 2016). Effectivement, nos discussions sur la participation ont mis en évidence les potentialités de la citoyenneté à l'échelle urbaine pour le dépassement des identités nationales, qui sont, elles, davantage contraintes par les catégories raciales, religieuses et ethniques. Ceci dit, il faudra dans l'avenir s'intéresser au savoir que recouvre notre mode local de participation citoyenne, et mieux comprendre comment cette façon de faire peut être perçue ou acquise à la suite de l'immigration.

\section{Conclusion}

La méthodologie décrite dans ce texte, même si elle n'a pas été validée à grande échelle, offre des possibilités intéressantes pour générer des données empiriques sur l'acte à poser en contexte interculturel. Nous avons vu également que les ateliers de situations interculturelles peuvent avoir un impact sur la compréhension des enjeux soulevés, notamment par le développement de nouvelles compétences (comme la capacité à distinguer une situation et une préoccupation, ou la capacité à faire une centration). Un effet inattendu des ateliers - mais très heureux étant donné les objectifs de la recherche - est le rapprochement entre différents acteurs et organismes concernés par les dynamiques interculturelles dans l'espace urbain. Cette activité permet en effet aux acteurs sur le terrain de voir la diversité d'approches et les angles d'analyse possibles au sein de différents milieux de pratiques, pour travailler dans une logique complémentaire apte à valoriser différentes formes d'expertise et de savoir. 
Le langage commun développé dans le cadre de l'atelier fait davantage ressortir les objectifs partagés ainsi que la nécessité d'une approche complémentariste pour atteindre ces objectifs (Devereux, 1980). Une chose est certaine : l'acte à poser en contexte interculturel exige de nouvelles conditions et de nouvelles compétences (Gratton, 2009; White, Gratton et Rocher, 2015). Ceci demande non seulement un travail sur un vocabulaire et un cadre de référence minimalement partagés, mais aussi la création de nouveaux outils aptes à décrire des situations en nous assurant que nous voyons tous plus ou moins la même chose (ce que Gadamer appelle le subjectum, voir Gadamer, 1996) avant de prendre une décision sur l'acte à poser. Des conditions particulières sont également nécessaires pour s'assurer qu'un acte posé par un professionnel (intervenant, gestionnaire, décideur, chercheur, etc.) reçoit le soutien de l'organisation et d'une institution qui se dit vouloir combattre l'exclusion des personnes et des groupes issus de l'immigration.

Un acte à poser, que ce soit un acte de professionnel ou un acte citoyen, n'est jamais une action individuelle isolée. II relève aussi des configurations organisationnelles et institutionnelles, elles-mêmes porteuses de traditions. II s'inscrit dans des mandats et des rôles, des privilèges et des responsabilités. II s'adresse à un public particulier avec des attentes particulières et il exprime le besoin et la capacité d'interpeler l'Autre (ce que Bakhtin appelle l'addressivité). Ce qui nous a surpris dans le cadre du travail sur les ateliers de situations interculturelles, c'est qu'un tel dispositif méthodologique puisse constituer en soi un acte à poser, car il a un impact sur la perception des participants mais aussi sur la qualité des données recueillies. Malgré tout ce que les critiques constructivistes nous ont appris sur le positionnement de nos rôles de chercheur dans la production du savoir, dans le cadre des ateliers de situations interculturelles, nous nous sommes retrouvés face à notre "façon d'intervenir " sur un système qui se mobilise au nom de l'inclusion des populations vulnérables et marginalisées, constatant que les intervenants sont le plus souvent laissés sans ressources et amenés à remettre en question la pertinence de leur expertise habituelle. Si nous avons été capables d'aborder ce sujet (l'acte à poser du chercheur) avec les participants dans les ateliers, c'est en partie parce que les objectifs et les outils de l'atelier ont émergé dans le cadre d'un projet de rechercheaction. Les ateliers de situations interculturelles nous offrent la possibilité de prendre connaissance d'une grande variété d'actions et de situations interculturelles, mais la méthodologie nous a aussi obligés à garder une certaine humilité par rapport à notre capacité de faire une analyse systématique des actes complexes à poser en contexte interculturel.

\section{Références bibliographiques}

Ahmed, S. (2012). On being included: racism and diversity in institutional life. Durham, NC: Duke University Press.

Amin, A. (2012). Land of strangers. Cambridge.

Bakhtin, M. (1993). Toward a philosophy of the act (édition de V. Liapunov et M. Holquist, trad. de V. Liapunov). Austin : University of Texas Press.

Bateson, G. et Reusch, J. (1951). Communication: The social matrix of psychiatry. New York : Norton.

Bathurst, L. (2015). Teaching Culture in Anthropology and Intercultural Communication. Conférence présentée au Congrès de l'American Anthropological Association, 18-22 novembre.

Bathurst, L. et La Brack, B. (2012). Anthropology, Intercultural Communication, and Study Abroad. Dans M. Vande Berg, M. Paige et K. Lou (dir.), Student learning abroad: what your students are learning, what they're not, and what you can do about it (p. 188-214). Sterling, VA : Stylus Publishing.

Battaglini, A. (dir.). (2010). Les services sociaux et de santé en contexte pluriethniques. Montréal : Éditions SaintMartin.

Bouchard, G. (2012). L'interculturalisme : un point de vue québécois. Montréal : Boréal.

Cantle, T. (2012). Interculturalism : The new era of cohesion and diversity. London : Palgrave.

Cohen-Émérique, M. (1993). L’approche interculturelle dans le processus d'aide. Santé mentale au Québec, 18(1), 71-91. 
Cohen-Émérique, M. (2011). Pour une approche interculturelle en travail social. Théories et pratiques. Rennes : Presses de l'EHESP.

Côté, D., Frozzini, J. et Gratton, D. (2013). La compétence interculturelle dans le contexte des services de réadaptation physique et des agences de placement temporaire au Québec. Revue S\&F, avoirs et formations, recherches et pratiques, 3, 78-93.

Das, K. (1993). Formation présentée à I'Institut Interculturel de Montréal [contenu inédit]. Montréal : Institut Interculturel de Montréal.

Devereux, G. (1980). De l'angoisse à la méthode dans les sciences du comportement. Paris : Flammarion.

Emongo, L. et White B.W. (dir.). (2014). L'interculturel au Québec. Rencontres historiques et enjeux politiques. Montréal : Presses de l'Université de Montréal.

Emongo, L. (2014). Introduction à une épistémologie de l'inter-cultures. Dans L. Emongo et B. White (dir.), L'interculturel au Québec. Rencontres historiques et enjeux politiques (p. 221-250). Montréal, Presses de I’Université de Montréal.

Fourot, A.C. (2013). L'intégration des immigrants : cinquante ans d'action publique locale. Montréal : Presses de I’Université de Montréal.

Frozzini, J. et Gratton, D. (2015). Travail migrant temporaire et précarisation. Vie économique, 7(1), 1-10.

Gadamer, H.-G. (1996). Vérité et méthode : les grandes lignes d'une herméneutique philosophique. Paris : Seuil.

Gratton, D. (2009). L'interculturel pour tous : Une initiation à la communication du troisième millénaire. Montréal : Éditions Saint Martin.

Gratton, D. (2013). Préoccupations et attentes en réadaptation physique dans des contextes pluralistes : vers un cadre théorique interculturel. Thèse de doctorat, Département d'anthropologie, Université de Montréal.

Labelle, M. (2010). Racisme et anti-racisme au Québec : Discours et déclinaisons. Montréal : Presses de l'Université du Québec.

Legault, G. et Lafrenière, M. (1992). Situations d'incompréhensions interculturelles dans les services sociaux : problématique. Santé mentale au Québec, 17(2), 113-131.

Maalouf, A. (1998). Les identités meurtrières. Paris : Grasset.

Martiniello, M. (2011). La démocratie multiculturelle. Paris : Presses de Sciences Po.

Nail, T. (2015). The figure of the migrant. Stanford University Press.

Proulx, J. (1998). Gestion de la diversité en contexte d'une approche clientèle et d'une gestion des ressources humaines axées sur les résultats (vol. 1). Québec : Ministère de la Santé et des services sociaux du Québec.

Rocher, F. et B. W. White. (2014). L'interculturalisme québécois en contexte multiculturel canadien : Origines, critiques et politiques publiques. Montréal : Institut de recherches en politiques publiques.

Roy, G. (1992). Devons-nous avoir peur de l'interculturel institutionnalisé ? Nouvelles pratiques sociales, 5(5), 5364.

Vertovec, S. (2007). Super-diversity and its implications, Ethnic and racial studies, 30(6), 1024-1054.

White, B. W. (2014). Quel métier pour l'interculturalisme? Dans L. Emongo et B. White (dir.), L'interculturel au Québec. Rencontres historiques et enjeux politiques (p. 21-44). Montréal : Presses de l’Université de Montréal.

White, B. W. (2016). Le vivre-ensemble comme scénario de l'interculturel au Québec. Dans F. Saillant (dir.), Pluralité et vivre-ensemble (p. 39-62). Québec : Presses de l'Université Laval.

White, B. W. (2017a). Jamais deux sans trois : La situation ethnographique et le rapport à l'autre. Dans M. Lantin, M. Blondet et A. Bensa (dir.), De la reflexivité à la vigilance. Lyon : Presses universitaires de Lyon. À paraître. 
White, B. W. (2017b). Pensée pluraliste dans la cité : L'action interculturelle à Montréal. Anthropologie et sociétés, automne, numéro spécial sur l'interculturel dans la Cité. À paraître.

White, B. W., Gratton, D. et Rocher, F. (2015). Les conditions de l'inclusion en contexte interculturel. Mémoire présenté à la Commission des relations avec les citoyens, Ministère de l'Immigration, de la Diversité et de l'Inclusion. 